\title{
Mechanism of hologram formation on glass surface by recording technique with corona discharge
}

\author{
Daisuke Kobayashi, ${ }^{1 *}$ Yuichi Yamamoto, ${ }^{2}$ Kiyoshi Yamamoto, ${ }^{1}$ Shiro Funatsu, ${ }^{3}$ Kenji Harada ${ }^{4}$ and Junji Nishii ${ }^{5}$ \\ ${ }^{1}$ Research center, ASAHI GLASS CO., LTD., 1150 Hazawa-cho, Kanagawa-ku, Yokohama, Kanagawa 221-8755 Japan \\ ${ }^{2}$ AGC ELECTRONICS CO., LTD., 1-8 Machiikedai, Koriyama, Fukushima 963-0215 Japan \\ ${ }^{3}$ AGC GLASS EUROPE, Rue de l'Aurore 2 - B-6040 Jumet Belgium \\ ${ }^{4}$ Department of Computer Science, Kitami Institute of Technology, 165 Koen-cho, Kitami, Hokkaido 090-8507 Japan \\ ${ }^{5}$ Research Institute for Electronic Science, Hokkaido University, Kita 21 Nishi 10, Kita-ku, Sapporo 001-0021 Japan \\ *daisuke-kobayashi@agc.com
}

(Received : November 18, 2013; Accepted : January 9, 2014)

\begin{abstract}
Surface relief grating of azobenzene polymer formed on a soda-lime-silica glass plate was treated by corona discharge for the formation of a hologram on the glass plate. The Na concentration distribution near the glass surface was investigated using a time-of-flight secondary ion mass spectrometry (TOF-SIMS) depth analysis with buckminsterfullerene $\left(\mathrm{C}_{60}\right)$ ion sputtering and an image processing. The periodical $\mathrm{Na}$ deficient region identical to the azobenzene grating pattern existed near the glass surface, which suggested that the $\mathrm{Na}$ ions below the grating groove preferentially migrated to the cathode side during the corona discharge treatment. Generally, the refractive index of the Na poor region becomes lower than that of the pristine soda-lime silica glass. Therefore, the origin of the hologram on the glass surface should be the three-dimensional distribution of refractive index at the glass surface.
\end{abstract}

\section{Introduction}

Formation of three-dimensional holograms inside glasses is a key technology for the next generation functional glass products. Multi-photon processing using femtosecond laser [1] or visible radiation absorbents are required for the hologram recording owing to a high optical transparency of glass. Recently, it was found that a surface-relief hologram of an azobenzene polymer film on a soda-lime-silica glass plate was recorded on the glass surface by the use of corona discharge process [2-6]. When the corona discharge treatment is conducted on the glass plate without the polymer film, sodium poor and proton rich layer is formed on the glass surface [7]. Therefore, in order to understand the mechanism of hologram formation on the glass with the polymer film, it is essential to investigate the cross-sectional or three-dimensional concentration distribution of sodium near the glass surface.

Generally, precise depth profile of alkali metal elements, such as sodium, in insulators cannot be obtained by surface analysis with ion sputtering because the ele- ments migrate from the sample surface to the inside during sputtering process $[8,9]$. The migration of the elements is induced by the formation of an electric field and the increase of the sample temperature. Recently, the precise depth profile of sodium in glass has been successfully acquired by the use of buckminsterfullerene $\left(\mathrm{C}_{60}\right)$ ion sputtering $[10,11]$. Depth profile analysis by time-of-flight secondary ion mass spectrometry (ToF-SIMS) is usually performed in a dual beam mode alternately using a pulsed primary ion beam for analysis in the static regime and a continuous ion beam for sputtering. It has been confirmed that the sodium in $\mathrm{SiO}_{2}$ glass does not migrate during both the analysis process with Bi primary ion beam and the sputtering process with $\mathrm{C}_{60}$ ion beam [11]. TOF-SIMS has superior spatial resolution and can acquire an elemental mapping on surface. Therefore, cross-sectional and three-dimensional elemental mapping is expected to be obtained by TOF-SIMS depth analysis with $\mathrm{C}_{60}$ ion sputtering and by image processing. In this study, to understand the mechanism of hologram formation by corona discharge, the 


\section{Kobayashi et al. Mechanism of hologram formation on glass surface by recording technique with corona discharge}

cross-sectional concentration distribution of sodium near the glass surface is investigated by TOF-SIMS depth analysis with $\mathrm{C}_{60}$ ion sputtering.

\section{Experimental}

Azobenzene polymer (poly-orange tom 1) was dissolved in cyclohexanone at $10 \mathrm{wt} \%$ and spin-coated on a soda-lime silica glass plate (S-7213, Matsunami Glass Ind., Ltd.). Then the sample was baked on a hot plate at $140{ }^{\circ} \mathrm{C}$ for $10 \mathrm{~min}$. The thickness of the polymer and the glass plate were approximately $1 \mu \mathrm{m}$ and $1 \mathrm{~mm}$, respectively. To form surface relief grating on the polymer, the sample was exposed to collimated $\mathrm{Ar}^{+}$laser irradiation at wavelength of $514 \mathrm{~nm}$ for $30 \mathrm{~min}$. The grating period was $4 \mu \mathrm{m}$.

A cross section image of the sample was observed using a scanning electron microscope (SEM) to confirm the surface relief structure of the azobenzene polymer. The SEM observation was conducted by a S-3000N (Hitachi Ltd.) at an acceleration voltage of $15 \mathrm{kV}$. Carbon was coated on the sample before the SEM observation to avoid charging. TOF-SIMS depth analysis with $\mathrm{C}_{60}$ sputtering was carried out on a TOF-SIMS ${ }^{5}$ (ION-TOF $\mathrm{GmbH})$. In this work, the following conditions were used: $\mathrm{Bi}_{3}{ }^{++}$primary ion $(25 \mathrm{kV}), 0.03 \mathrm{pA}$ at $10 \mathrm{kHz}$ beam current, primary ion in long pulse mode (burst alignment mode), $100 \mu$ s cycle time, $19.5 \times 19.5 \mu \mathrm{m}^{2}$ region of interest, $\mathrm{O}_{2}$ flooding $\left(5 \times 10^{-6} \mathrm{mbar}\right.$ vacuum $)$ and room temperature. The depth profiles of the sample were acquired by $\mathrm{C}_{60}{ }^{++}$ion sputtering $(10 \mathrm{kV})$ in the non-interlaced mode (longer sputter and data acquisition cycles). Two different rate sputtering conditions were employed to clarify the whole picture of concentration distribution of sodium near the glass surface. Low-rate sputtering was conducted by $1.0 \mathrm{nA}$ at $10 \mathrm{kHz}$ beam current and $400 \times 400 \mu \mathrm{m}^{2}$ raster size, while high-rate sputtering by $1.5 \mathrm{nA}$ at $10 \mathrm{kHz}$ beam current and $150 \times 150$ $\mu \mathrm{m}^{2}$ raster size. Effective charge compensation was realized by using an electron flood gun $(20 \mathrm{eV})$. Three-dimensional spatial data including information of mass spectra was obtained by TOF-SIMS depth analysis. Cross section images of $\mathrm{C}_{6} \mathrm{H}_{5} \mathrm{~N}^{+}, \mathrm{Na}^{+}$and $\mathrm{Si}^{+}$were constructed from the three-dimensional spatial data by image processing.

\section{Results and discussion}

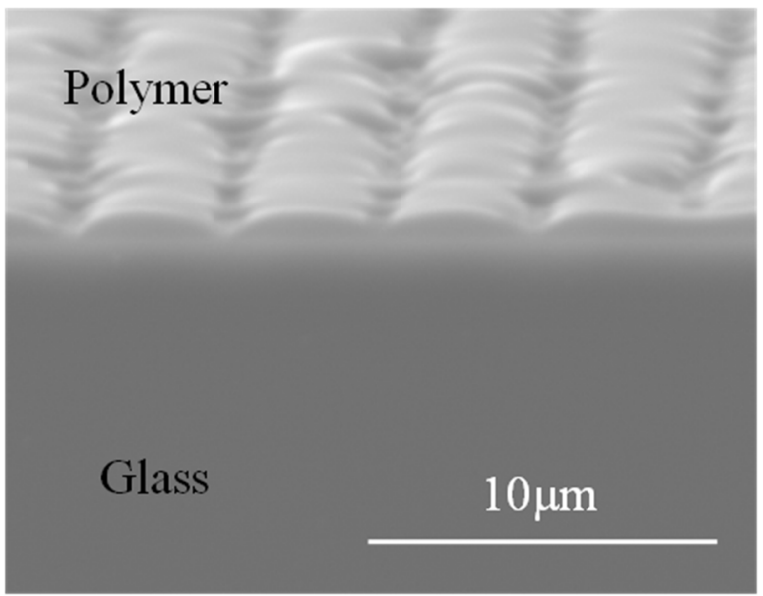

Fig. 1 A cross-sectional SEM image of azobenzene polymer with surface relief grating on soda-lime-silica glass. The height and the grating period of the polymer are $1 \mu \mathrm{m}$ and $4 \mu \mathrm{m}$, respectively.

Figure 1 shows a cross-sectional SEM image of the sample. It is confirmed that the height and the grating period of the polymer are $1 \mu \mathrm{m}$ and $4 \mu \mathrm{m}$, respectively.

Figure 2 shows cross-sectional TOF-SIMS images of $\mathrm{C}_{6} \mathrm{H}_{5} \mathrm{~N}^{+}, \mathrm{Na}^{+}$and $\mathrm{Si}^{+} . \mathrm{C}_{6} \mathrm{H}_{5} \mathrm{~N}^{+}$is originated from azobenzene polymer. $\mathrm{Na}^{+}$and $\mathrm{Si}^{+}$are from soda-lime-silica glass. Upper and lower images were obtained by low and high rate conditions of $\mathrm{C}_{60}$ ion sputtering, respectively. In the TOF-SIMS image, the sample surface is flatly described. Scale of depth direction is not the real depth but the sputtering time. Since sputtering rate of the polymer is considerably higher than that of the glass, the thickness of the polymer is underestimated. $\mathrm{Na}$ poor region exists near the glass surface and has periodicity which is the same period as surface relief grating of the polymer, $4 \mu \mathrm{m}$. The bottom edge of the Na poor region corresponds to the valley of the polymer. The depth of the bottom edge was estimated to be $0.45 \mu \mathrm{m}$ provided the $\mathrm{C}_{60}$ ion sputtering rate of soda-lime-silica glass was employed. Meanwhile, intensity of $\mathrm{Si}^{+}$is higher at the $\mathrm{Na}$ poor region than at the inner side of the glass. This phenomenon is assumed to be caused by the matrix effect associated with formation of the $\mathrm{Na}$ poor region.

The mechanism of the hologram formation on the glass surface by corona discharge is illustrated in Fig. 3. The polymer surface is charged positively by corona discharge. The glass plate is relatively affected by the electric fields at valley compared with that at top of the grating. Below the valley of the grating, $\mathrm{Na}^{+}$migrates 


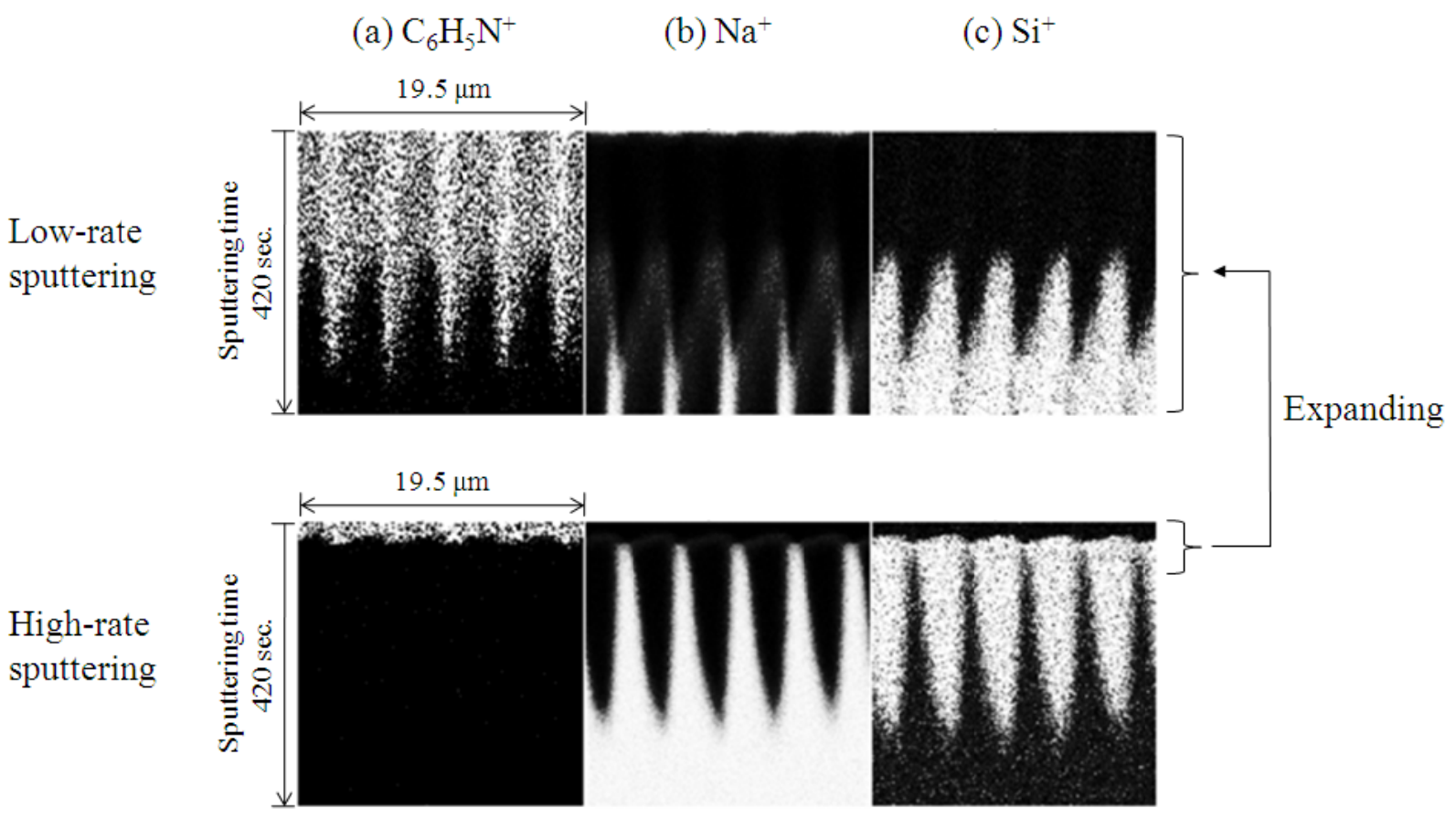

Fig. 2 Cross-sectional TOF-SIMS images of (a) $\mathrm{C}_{6} \mathrm{H}_{5} \mathrm{~N}^{+}$, (b) $\mathrm{Na}^{+}$and (c) $\mathrm{Si}^{+} . \mathrm{C}_{6} \mathrm{H}_{5} \mathrm{~N}^{+}$is originated from azobenzene polymer. $\mathrm{Na}^{+}$and $\mathrm{Si}^{+}$are from soda-lime-silica glass. Upper and lower images were obtained by low and high rate conditions of $\mathrm{C}_{60}$ ion sputtering, respectively.

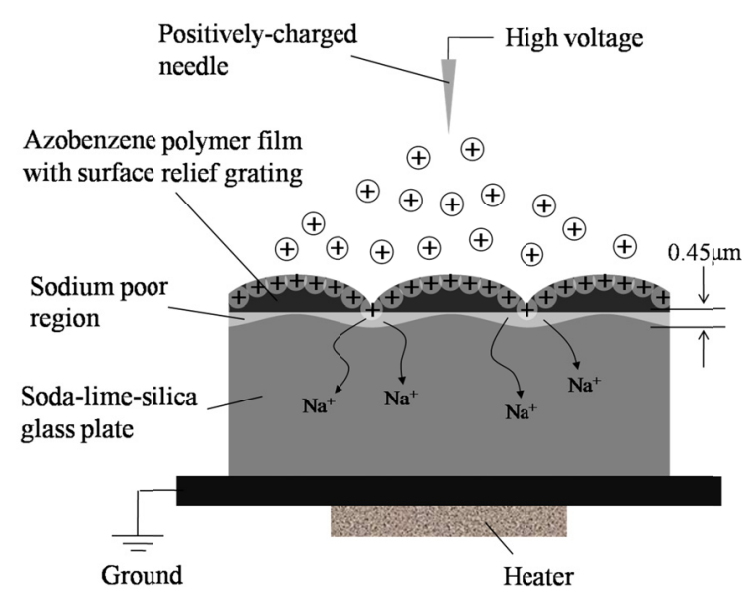

Fig. 3 Mechanism of the hologram formation on soda-lime-silica glass surface using corona discharge.

easily from surface to inside. Generally, the refractive index of Na poor region is lower than that of the pristine glass. The origin of the hologram is the three-dimensional distribution of refractive index at the glass surface. The surface relief structure of the polymer can be copied on the glass surface.

\section{Conclusion}

Corona discharge treated the surface relief grating of an azobenzene polymer on a soda-lime-silica glass plate. The concentration distribution of sodium near the glass surface could be confirmed using a TOF-SIMS depth analysis with $\mathrm{C}_{60}$ ion sputtering and an image processing. $\mathrm{Na}$ poor region formed near the glass surface has the periodicity identical to the surface relief grating period of the polymer. The origin of the hologram on the glass is the three-dimensional distribution of refractive index at the glass surface, because the refractive index of Na poor region is lower than that of the pristine glass. Therefore, the corona discharge treatment is useful to transfer a surface relief structure on the polymer to an alkali containing glass plate.

\section{References}

[1] S. Juodkazis, A. V. Rode, E. G. Gamaly, S. Matsuo and H. Misawa, Appl. Phys. B 77, 361 (2003).

[2] D. Sakai, K. Harada, S. Kamemaru and T. Fukuda, Appl. Phys. Lett. 90, 061102 (2007).

[3] D. Sakai, K. Harada, S. Kamemaru, D. Barada, F. 
Journal of Surface Analysis Vol.20, No. 3 (2014) pp. 226-229

D. Kobayashi et al. Mechanism of hologram formation on glass surface by recording technique with corona discharge

Sato and T. Fukuda, Jpn. J. Appl. Phys. Part 1 47, 7929 (2008).

[4] D. Sakai, D. Miho, K. Harada, D. Barada and T. Fukuda, Jpn. J. Appl. Phys. Part 1, 49, 01AE01 (2010).

[5] D. Sakai, K. Harada, S. Kamemaru, D. Barada, F. Sato and T. Fukuda, Opt. Rev. 16, 335 (2009).

[6] D. Sakai, K. Harada, S. Kamemaru, and T. Fukuda, Opt. Rev. 14, 339 (2007).

[7] H. Ikeda, D. Sakai, S. Funatsu, K. Yamamoto, T. Su- zuki, K. Harada and J. Nishii, J. Appl. Phys. 114, 063303 (2013).

[8] H. L. Hughes, R. D. Baxter and B. Phillips, IEEE Trans. Nuci. Sci. NS-19, 256 (1972).

[9] Y. Hayashi, K. Matsumoto, Bunseki Kagaku 42, 243 (1993).

[10] Y. Yamamoto and K. Yamamoto, J. Non-Cryst. Solids 356, 14 (2010).

[11] D. Kobayashi, Y. Yamamoto and T. Isemura, Surf. Interface Ana1.45, 113 (2013). 